\title{
Towards Shorter Wavelength X-ray Lasers using a High Power. Short Pulse Pump Laser
}

PPPL $=-2753$

DE91 012306

W. Tighe, K. Krushelnick, E. Valeo, and S. Suckewer a

Princeton University Plasma Physics Laboratory, Forrestal Campus, P.O. Box 451

Princeton, N.J., U.S.A., 08544

\begin{abstract}
A near-terawatt, $\mathrm{KrF}^{*}$ laser system, focussable to power densities $>10^{13} \mathrm{~W} / \mathrm{cm}^{2}$ has been constructed for use as a pump laser in various schemes aimed at the development of $X$-ray lasing below $5 \mathrm{~nm}$. The laser system along with output characteristics such as the pulse duration, the focal spot size, and the percentage of amplified spontaneous emission (ASE) emitted along with the laser pulse will be presented. Schemes intended to lead to shorter wavelength $X$-ray emission will be described. The resultant requirements on the pump laser characteristics and the target design will be outlined. Results from recent solid target experiments and two-laser experiments, showing the interaction of a high-power, short pulse laser with a preformed plasma, will be presented.
\end{abstract}

\section{INTRODUCTION}

The field of $X$-ray lasers has developed rapidly in the past decade (e.g. reviews by Skinner, 1990, Suckewer and Skinner, 1990 , and Matthews, 1990). Several groups now have active programs addressing application of these devices (e.g. Suckewer, $1990, \mathrm{Kim}, 1990$ and Trebes, 1990 ). Areas of interest include cell microscopy, holographic imaging of biological material, $x$-ray litiography, micro-chip fabrication and inspection, and high density plasma diagnostics among others. These applications can benefit from operation at shorter wavelength since this provides better resolution and greater penetration. While much progress has been made in demonstrating $x$-ray lasing near and in the so-called water window (2.4-4.4 nm), the techniques used to date still require the use of the worlds largest lasers to pump the transitions. The purpose of our research is to investigate more efficient and practical approaches to the generation of shorter wavelength $x$-ray lasers and in this report we present data marking our progress in this area.
To examine approaches to the development of shorter wavelength $X$-ray lasers one is primarily concerned with the requirements placed on the pump source. To maintain a specific gain-length characteristic of a lasing medism, the wavelength scaling of the pump power is as severe as $P \propto \lambda^{-4}$ so that a decrease of a factor of 10 in operating wavelength implies an increase in pump power of over 4 orders of magnitude. For shorter wavelength, the transitions being pumped are also very short-lived and the pumping time can be significantly reduced. In fact for efficient pumping these time scales should be closely matched. To generate the high power required, it is far more reasonable to incorporate a short pulse (subpicosecond) pump laser (see Schwarzenbs.ch et al., 1986, Tighe et al., 1990 and references therein). Still further increase in pump intensity is possible by using an ultraviolet pump source since this has better focusability and would utilize lower order non-linear processes which are likely to be invoked in the absorption mechanism. This line of reasoning led us to develop the Powerful SubPicosecond (PSP) laser operating at the $\mathrm{KrF}^{*}$ wavelength of $248 \mathrm{~nm}$. In developing ideal conditions for

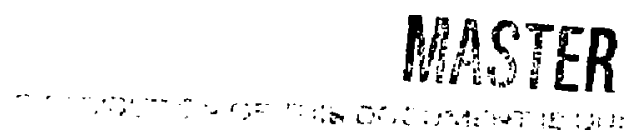




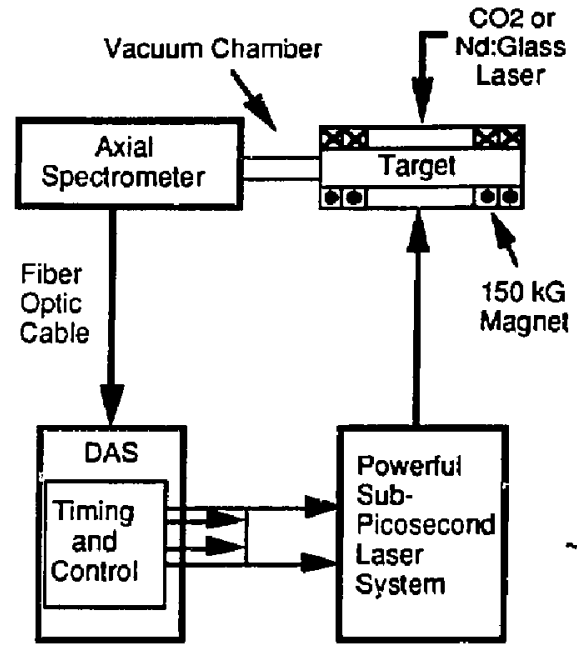

(a)

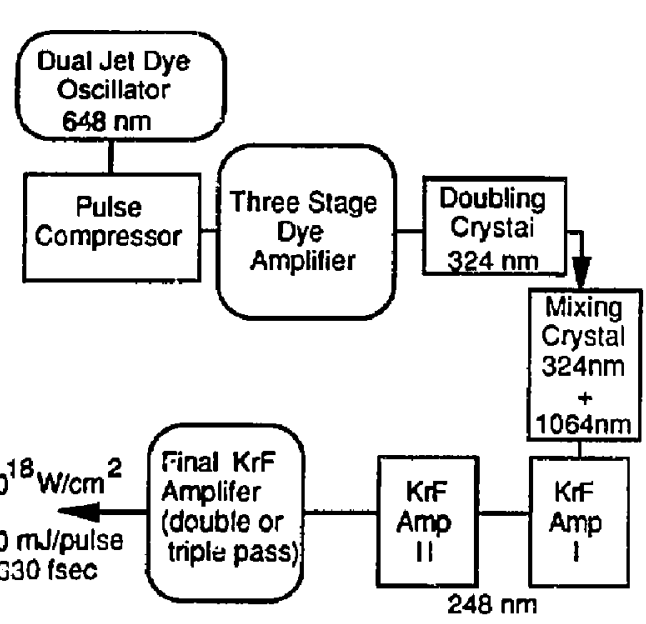

(b)

Fig.1. a) Block diagram of Two-Laser Approach. b) Block diagram of Powerful Subpicosecond (PSP) Laser system.

gain at shorter $X$-ray wavelengths, two approaches can be considered. In one case a high temperature plasma is created by a single laser. If conditions of high density and rapid cooling are put into place then three-body recombination and cascade processes will allow upper levels to be populated while levels near the ground state will be rapidly, radiatively depopulated. Population inversions and gain are possible. By selecting appropriate elements this recombination scheme may be effectively scaled to shorter wavelengths. An altemative technique is to obtain the population inversion without heating the plasma (see Burnett and Corkum,1988). In this case the plasma state would be preformed and once the desired ionization stage was reached, a high power laser would be used to excite or further ionize the ion by a multiphoton process and, thereby, create the inversion. Because there is no significant heating of the free electron population the conditions for gain are more readily attainable. This approach is referred to as the "Two-Laser Approach" since the high ionization states can be rtadily obtained with a long pulse, high energy laser and the inversion condition can be best delivered with a high power, short pulse laser. A basic block diagram showing the primary elements of such a twolaser approach is given in figure 1 a). This experiment is now in place with critical issues of timing jitter and target alignment resolved. Some initial results will be presented later.

\section{SHORT PULSE LASER SYSTEM}

The PSP-Laser is a critical element of both approaches to short wavelength $X$-ray lasing. In the two-laser approach high-order muitiphoton processes requiring extremely high power pumps are used. In scaling the recombination laser, the cooling rate must be faster than the recombination rate which at shorter wavelength transitions is on the order of several picoseconds so that the high density plasmas formed must not be heated at later times by the laser. The goal in the development of the PSP-laser was an output on the order of a terawatt, focussable to $>10^{18} \mathrm{~W} / \mathrm{cm}^{2}$. A block diagram of the system is shown in figure $1 \mathrm{~b}$ ) and a brief description follows.

The system begins with a 1 psec dye oscillator tuned to $648 \mathrm{~nm}$. A fiber-grating combination is used to compress these pulses to $200 \mathrm{fsec}$. This output is amplified in a 


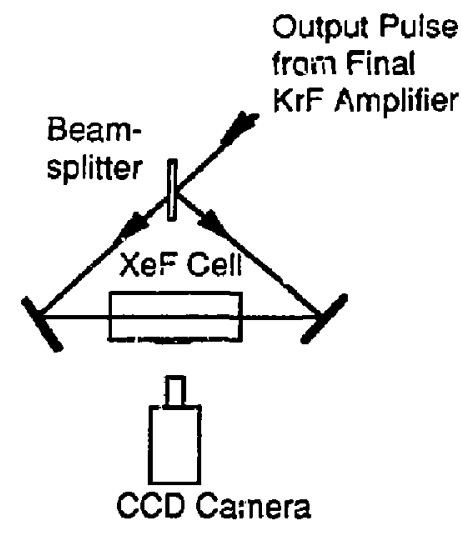

(a)

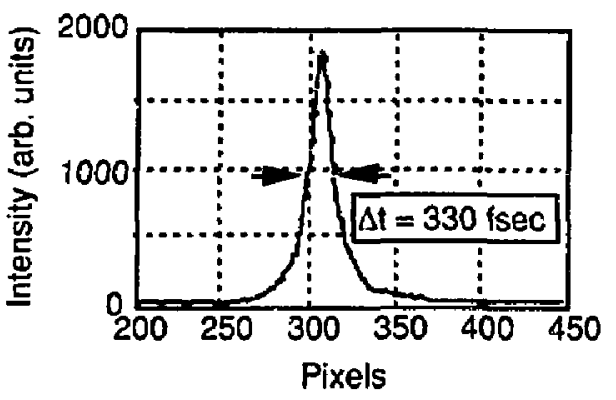

(b)

Fig. 2 a) Experimental set-up of single-shot autocomelator at $248 \mathrm{~nm}$. b) Duration of output pulse from PSP-Iaser

three-stage dye amplifier to a level of $\sim 100$ $\mu J$ per pulse and then frequency doubled (324 nm) in a $1 \mathrm{~mm}$ BBO crystal. This is mixed in a $1 \mathrm{~mm}$ KDP crystal with $1.06 \mu \mathrm{m}$ laser radiation to produce a seed pulse at 248 nm. Finally, this pulse is injected into a $\mathrm{KrF}^{*}$ amplifier chain.

Each $\mathrm{KrF}^{*}$ amplifier is separated by more than 20 nsec to prevent self oscillation and both spatial filtering and saturable absorbers are used between them in order to reduce the level of amplified spontaneous emiss!on (ASE). The final amplifier was constructed at PPPL (Meixler et al., 1988) and has an aperture of $5 \times 10 \mathrm{~cm}$ and a discharge length of $80 \mathrm{~cm}$. The system has been operated in either a double or a triple-pass mode. In the double-pass mode a single pulse is amplified to a level of $2200 \mathrm{~mJ}$ with a $10 \%$ ASE component. In íriple pass a multiplex scheme was invoked which allowed a train of pulses separated by -4 ns to be injected. This temporal separatior corresponds to the gain recovery time in the amplifier and appears to very effectively reduce the ASE component. The energy per pulse from this arrangement is on the order of $100 \mathrm{~mJ}$. Attempts are being made to multiplex angularly and then de-muitiplex these pulses. At present, all pulses are collinear and brought on target with the 4 nsec separation in time.
In determining the focal spot intensity, the important output characteristics of the laser system are the pulse duration and the focal spot size. To measure the pulse duration a single shot autocorrelator was developed which makes use of a three-photon fluoresence process in a XeF cell (Sarakura et al., 1988). A schematic of the autocorrelator and the corresponding measurement are shown in figure $2 \mathrm{a}$ ) and $b$ ). While a certain amount of pulse broadening is observed, with energy measurements of $100-150 \mathrm{~mJ}$ per pulse, the output power of the system is still in the order of $.3-.5$ terawatts. A single shot measurement of the focal spot size was also made. In this case a small fraction $(.04 \%)$ of the laser output was brought to a focus with a spherically and chromatically corrected $\mathrm{f} / 3 \quad \mathrm{MgF}_{2}$ lens system. The focal spot was re-imaged (magnified 15X) using a reflecting microscope objective onto a conversion plate. This was then examined using a $2 \mathrm{D} C \mathrm{CD}$ array camera yielding a total magn.ification of $\sim 50 \mathrm{X}$. Because misalignment will jroduce a larger than actual value, our measurement indicates focal spot dimensions of less than $4 \times 10 \mu \mathrm{m}$. Using these measurements the focal spot iniensity is determined to be $\sim 2 \mathrm{x}$ $10^{18} \mathrm{~W} / \mathrm{cm}^{2}$.

Another important characteristic of laser pulses of this type is the existence of prepuls= energy. In this system ASE can become very significant and much care has to 
be taken to control it. Because it is expected to play only a minor role in the two-laser experiment and become critical only in solid target interaction, we will discuss this feature in a later section.

\section{TWO-LASER APPROACH}

The essential aspect to the two-laser approach involves the interaction of an intense eiectric field (associated with a laser pulse) with a preformed ion. The ion will generally be highly charged and the role of the electric field is either to excite or remove the "next" electron in the ion. Several theoretical approaches to these problems have been initiated. With respect to excitation, the experimental control required, to utilize what appear to be extremely narrow resonances, make this approach difficult. The approach has not been abandoned since there are many factors which may assist by providing some unanticipated control. A more encouraging approach involves the further ionization of the target plasma with subsequent recombination leading to population inversions and gain. To simulate this process a Thomas-Fermi model (Susskind et al., 1991) was developed. A brief outline of this model follows.

The Thomas-Fermi model retains the screening effect of inner shell electrons in a many-electron atom by treating all the bound electrons as a fermi gas at zero temperature. Since the laser frequency $\omega$ is small compared to the atomic transition rate, $\omega<<$ the applied field can be treated as a constant. The ionization state of the atom will be determined by the maximum value of the applied field. The atom is filled with electrons with momenta,

$$
\mathrm{p} \leq \mathrm{p}_{\max } \equiv[2(\phi-\mathrm{W}+\mathrm{Fz})]^{1 / 2}
$$

where $W$ is the kinetic energy, and where $F$ is the maximum value with $\mathrm{z}$ as the direction of the applied field. This allows the electron density to be determined and using Poisson's equation a nonlinear, ordinary, integrodifferential equation in two dimensions (using azimuthal symmetry) for the selfconsistent potential $\phi$ is obtained. The description is completed with appropriate boundary conditions. With a change of variables the description is $\mathrm{Z}$-independent allowirg $\rightarrow$ singls solution to be applied to different elements. The model compares very well with experimental data as indicated in fig. 4 of Susskind et al. (1991).

Two points should be noted. First, there are no adjustable parameters in fitting this data, so the essential physics can be assumed to be captured in this very simple model. Second, what has been neglected in this simple presentation is the qliantum tunnelling, which can be shown, in a more complete calculation, to be negligible.

Initial experiments in the two-laser approach have been performed. The geometry is shown in figure 3. A thin blade target of either aluminum or teflon was used. A small ( $300 \mu \mathrm{m}$ diameter) alignment pinhole was placed near the edge of the target. A 3 nsec, $-10 \mathrm{~J}, \mathrm{YAG}$ :Glass laser pulse was brought on target to the alignment pinhole from one direction. The PSP laser pulse, focussed with a simple lens at $f / 10$, was brought from the opposite direction. The alignment method ensures that the lasers are focussed to the same location. The target is then lowered to a position where the thickness of the blade is significantly less than that through which the $1 \mu \mathrm{m}$ laser can bum. A transmission grating (in the spectral region 150-200 $\mathrm{A}$ ) is placed perpendicular to the interaction region and the a $50 \mathrm{~ns}$ streak of the spectra is obtained with a soft $x$-ray streak camera. The long duration plasma emission produced by the interaction of the long pulse laser is clearly seen on the streak photograph (not shown here). The time of onset of the shor pulse PSP laser is also clear - indicated by enhanced emission across the spectral region. While there is some indication of some spectral variation induced by the PSP laser pulse, detailed analysis of these results is pending the experimental modifications discussed below.

The major difficulties with the experimental arrangement described above are, first, the f/number does not provide the highest power from the PSP laser and, secondly, the required vaporization of the target by the YAG:Glass laser is not a particularly reliable technique and leaves open the possibility that the PSP laser interacts with resrnant solid rather than the produced plasma. Modification to the systemi are being made now in order to use the corrected $f / 3$ focussing optic for the PSP laser and to bring 


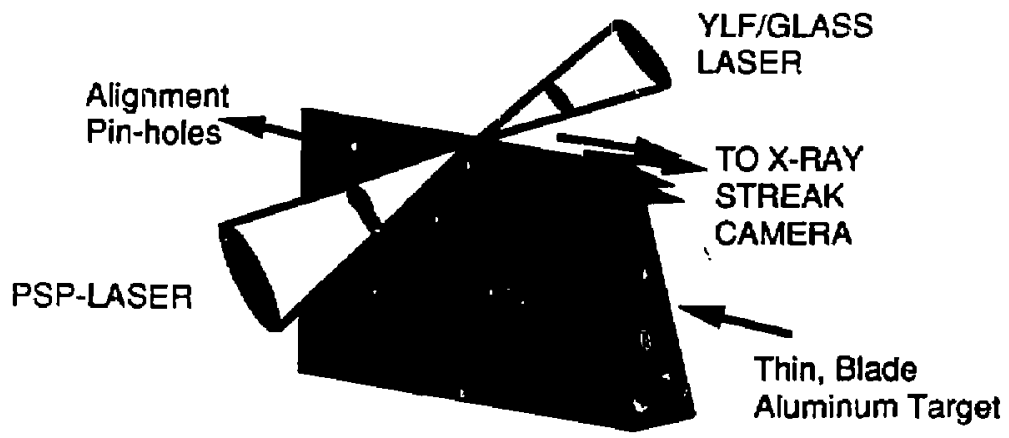

Fig. 3 Target geometry used in initial "two-laser approach" experiments

the laser on target perpendicularly to the first laser.

\section{SCALING THE RECOMBI- NATION X-RAY LASER}

It seems to be feasible to scale successful recombination laser experiments at $18.2 \mathrm{~nm}$ in carbon to higher $\mathbf{Z}$ elements and shorter wavelength transitions (Tighe et al., 1990). One possibility is the $\mathrm{H}_{\alpha}$ line in hydrogenlike aluminum at $3.9 \mathrm{~nm}$. Designs and sensitivity to laser parameters are discussed below.

The optimal conditions for recombination pumped gain are that the plasma density be as high as possible without collisionally quenching the lasing transition. The requirements on the electron temperature $T_{e}$ are that it change from a value comparable to the ionization potential $E_{i o n}$ to a small fraction thereof in a time short compared to the recombination time. In the case of $\mathrm{H}$ like aluminum the optimum density is of the order $\mathrm{n}_{\mathrm{e}}=10^{22} \mathrm{~cm}^{-3}$ and, with the threebody recombination rate being $\sim 2 . \cdot 10^{-12}$ $\mathrm{sec}$, a cooling time of a few psec is required if substantial gain is to be achieved.

The expected gair can be determined from the population inversion density. This can be estimated by assuming that each recombination event flows through the level of interest and by taking spontaneous emission as the dominant drain mechanism. The formula for the line-center gain ccefficient can then be expressed as,

$$
\kappa=\frac{\lambda_{u l}^{4}}{16 \pi^{2} c} \frac{n_{e}^{2} \alpha_{r e c}}{\Delta \lambda}
$$

The Balmer alpha transition wavelength in Aluminum is $\lambda_{32}=3.9 \mathrm{~nm}$. Using $\mathrm{n}_{\mathrm{e}}=8$ $.10^{21} \mathrm{~cm}^{-3}, \mathrm{n}_{\mathrm{e}} \alpha_{\mathrm{rec}}=4.10^{11} \mathrm{sec}^{-1}$ and $\Delta \lambda=$ $4 \cdot 10^{-3} \mathrm{~nm}$, we obtain a very large gain coefficient of, $\mathrm{K}=4 \cdot 10^{4} \mathrm{~cm}^{-1}$. The gain regions are expected to be of the order of microns so that gain lengths on the order of 10-20 might be expected.

We will now discuss some elements of the target design for a laser of this type. In a recombination time $\tau_{\text {rec, }}$ an impulsively heated, freely expanding plasma foil expands to a thickness $\Delta=C_{s} \tau_{r e c}$, where $\mathrm{C}_{S}$ is the sound speed determined by the electron temperature $\mathrm{T}_{\mathrm{e}}$ immediately after heating. For fully stripped Aiuminum, taking $\mathrm{T}_{\mathrm{e}}=700 \mathrm{ev}$, we have $\mathrm{C}_{\mathrm{S}}=1.8 \cdot 10^{7}$ $\mathrm{cm} \mathrm{sec}-1$. For $\tau_{\text {rec }}=2.5 \cdot 10^{-12} \mathrm{sec}$, we have $\Delta=4.5 \cdot 10^{-5} \mathrm{~cm}$. Assuming initially solid densities, which for fully stripped Al yields $\mathrm{n}_{\mathrm{e}, \mathrm{s}}=9 \cdot 10^{23} \mathrm{~cm}^{-3}$, the requirement that $n e<8 \cdot 10^{21} \mathrm{~cm}^{-3}$ yields an estimate for the thickness of the solid target of $\Delta_{S}=4$. $10^{-7} \mathrm{~cm}$.

In the approach described above, large temporal and spatial gradients are prerequisites fo: inversion between levels 
which decay via allowed transitions. This is the principal rationale for development of fsec pumping systems. In order to develop the required level of contrast between the pulse energy and the prepulse energy ( $\left.10^{4}\right)$, techniques such as spatial filtering and the use of saturable absorbers have been employed and while effective are generally limited to ratios of $\sim 10^{3}$. A "plasma saturable absorber" based on a thin film in a spatial filter configuration placed after the final amplifier may provide the required contrast.

\section{SOLID-TARGET INTERACTION}

In this section, spectra obtained from the interaction of the PSP laser pulse with solid slab targets will be presented, discussed and compared for different power intensities.In figure 4 spectra obtained from the interaction with a teflon target are presented. Figure 4 a), shows the result after 200 shots at a focal spot intensity of $10^{16} \mathrm{~W} / \mathrm{cm}^{2}$. The CVI and CV 2-1 transitions ( $33.7 \AA$ and $40.3 \AA$, respectively) are identified. There is some indication of the existence of satellite lines but they appear quite weak. Under conditions of higher focal spot intensity, in figure $4 \mathrm{~b}$ ), the number of shots have been reduced by a factor of 40 while the spectral intensity has decreased by approximately a factor of 2 . The lens used in obtaining these spectra was an uncorrected, simple lens with a focal length of $20 \mathrm{~cm}$. Most significantly, the satellite lines to the CVI and $C V$ transitions have become relatively much stronger. This indicates that either the emission is occurring under higher density conditions or that the line emission may coexist with the extremely high electric field of the laser. The line broadening of these lines is difficuls to use in determining the density due to the presence of satellite lines and
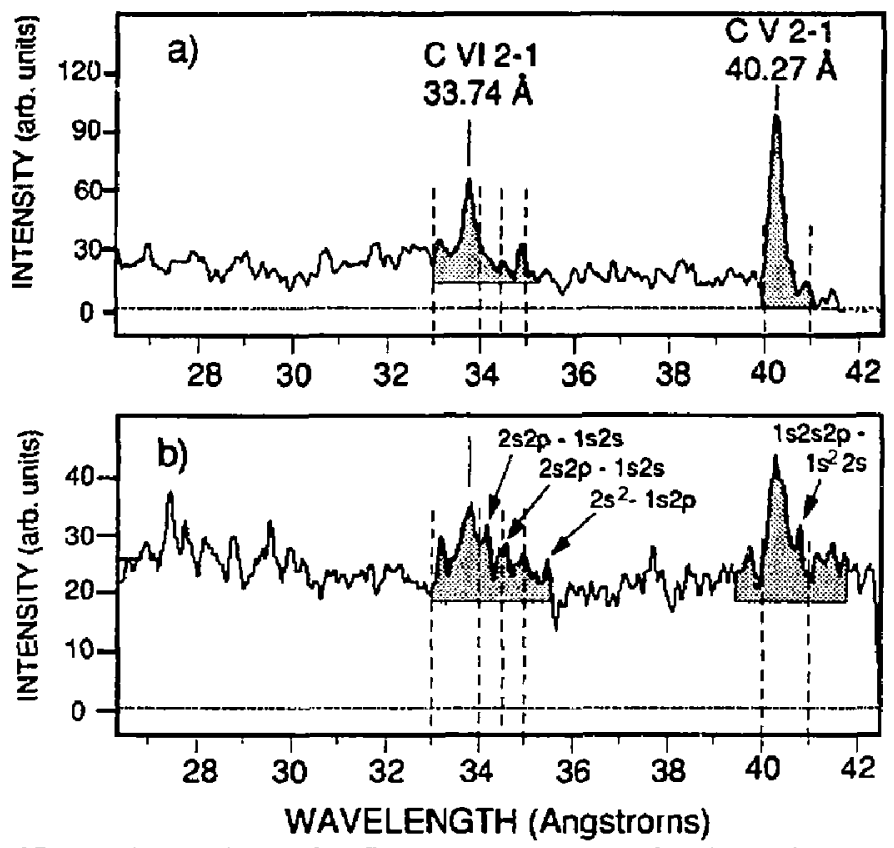

Fig. 4 PSP-laser interaction with teflon target. a) Accumulated over 200 laser shots at intensity of $\sim 2 \times 10^{16} \mathrm{~W} / \mathrm{cm}^{2}$.b) Accumulated over 5 laser shots at intensity of $-10^{17} \mathrm{~W} / \mathrm{cm}^{2}$. 
possible self absorption of the resonance emission.

With corrected, $f / 3$ focussing optics, the focal spot intensity on target is determined to be $2 \cdot 10^{18} \mathrm{~W} / \mathrm{cm}^{2}$. Spectra obtained from the interaction of this laser pulse with a teflon target are shown in figure 5 a). Similar to the results in figure 4 b), 5 shots were averaged to obtain this spectrum. Here, however, the signal intensity is larger by more than a factor of 2 . Moreover, the satellite lines are much more clearly identified. The CVI 3-1 transition is also apparent although there are no stgnificant satellite lines associated with it. Using the RATION code (Lee, 1990) a rough measure of the temperature and density from the ratios of these lines indicates that the line emission is occurring at near solid

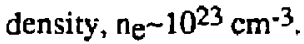

All the above spectra were taken under spatially and temporally integrated conditions. With certain orientations of the spectrometer a small degree of spatial resolution was obtained, allowing a view of the plasma closer to the target surface. The result, shown in figure 5 b), is marked by an extremely broad CVI 2-1 emission and no $\mathrm{CV}$ emission whatsoever. The implication here is that the emission is occurring under still higher density conditions. Although analysis of these spectra is not complete, additional broadening processes may be involved. A primary candidate would be the extremely high electric field associated with the laser. Temporally resolved spectra on the time scale of the laser pulse are necessary in order to clarify these results.

Finally, attempts have been made to observe the AI XIII 3-2 transition at $3.8 \mathrm{~nm}$. This was discussed earlier in terms of the scaling of the recombination laser to shorter wavelengths. Not shown here, the AI XIII 3-2 transition was observed though it was weak. This is not surprising since the density conditions are likely to exceed those at which the inversion is collisionally quenched. As discussed in the previous section, it is necessary to allow the Al plasma to expand to lower density prior to allowing it to cool rapidly. Apparatus to obtain these conditions is presently being constructed.
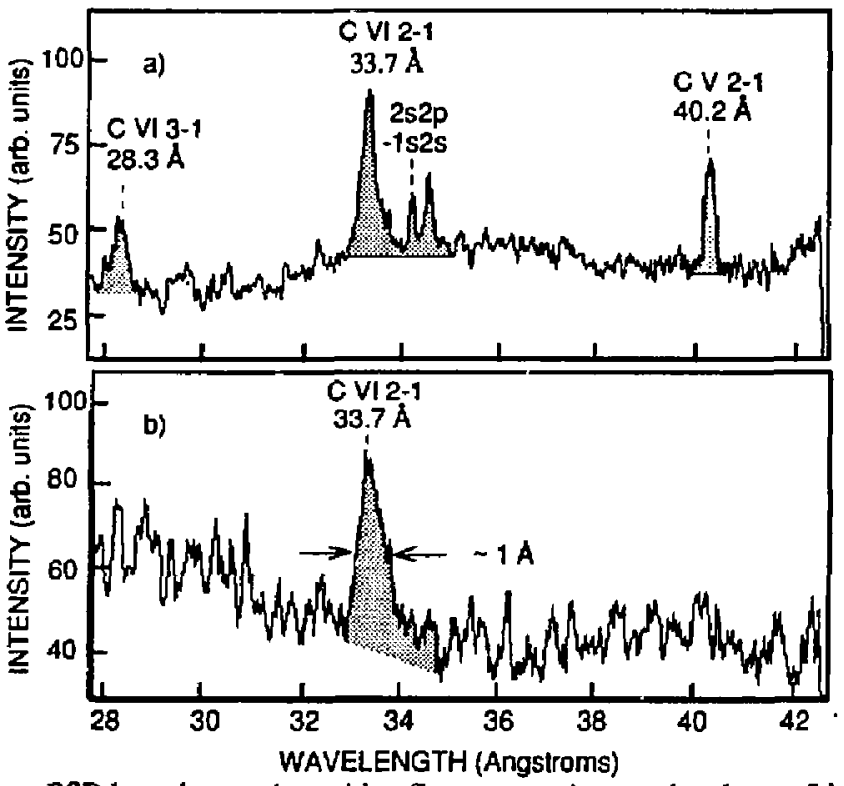

Fig. 5 PSP-laser interaction with teflon target. Accumulated over 5 laser shots at intensity of $\sim 2 \times 10^{18} \mathrm{~W} / \mathrm{cm}^{2}$. a) Spatially integrated spectra showing strong satellite lines b) Plasma viewed closer to target surface showing broadened line. 


\section{CONCLUSIONS AND \\ FUTURE WORK}

At present the PSP-Laser operates routinely jroducing single or multiple pulses on target with focal intensities in the order of $10^{18}$ $W / \mathrm{cm}^{2}$. We have observed high temperature, high density plasma conditions in solid target interaction studies and initiated low jitter $(\sim 1$ ns) two-laser experiments which indicate enhancement of plasma emission.

We are examining the possibility of scaling recombination laser to shorter wavelength using $\mathrm{H}$-like $\mathrm{Al}(39 \mathrm{~A})$. We are also redesigning the two-laser experiments to incorporate higher intensity laser interaction. Finally we hope to make use of two-laser experiments to investigate high harmonic generation in plasma.

\section{ACKNOWLEDGEMENTS}

The authors would like to acknowledge the help of J. Fujimoto (MIT) with the puise compression and J. Goldhar (Univ. of Maryland) with the development of the final $\mathrm{KrF}^{*}$ amplifier. The extensive technical support of J. Robinson was much appreciated. We further acknowledge the assistance of $\mathrm{L}$. Meixler and N. Tkach with engineering and electronic development.

This work was financially supported by the U.S. Department of Energy, Advanced Energy Projects of Basic Energy Sciences, Contract No. KC-05-01.

a Also with the Mecharical and Aerospace Engineering Department, Princeton Univ., Princeton, NJ.

\section{REFERENCES}

Burnett N. and Corkum P. B. $1989 \mathrm{~J}$. Opt. Soc. Am. B, 6, 1195

Kim D, Skinner C.H., Voorhees D., and Suckewer S., 1990 2nd International Colloquium, $X$-Ray Lasers, this proceedings.

Lee R., 1990 , LLNL, RATION code, private comm.,

Matthews D. 1990 2nd international Colloquium, X-Ray Lasers, this proceedings.

Meixler L., Nam C.H., Robinson J., Tighe W., Krushelnick K., Suckewer S., Goldhar J., 1989 Opt. Soc. Am. Proc., Short Wavelength Coherent Radiation: Generation and Applicasion, Vol. 2, (Falcone and Kirz, eds.), Cape Cod, p.

Sarakura N., Watanabe M., Endoh A., and Watanabe S., 1988,Opt. Lett., 13, 996

Schwarzenbach A.P., Luk T.S., McIntyre I.A., Johnson U., McPherson A. Boyer K., and Rhodes C.K., 1986, Opt. Lett., $2,499$.

Skinner C. 1990, review paper, Bull. Am. Phys. Soc., Plasma Physics Meeting, Cincinatti, submitted to Phys. Fluids

Suckewer S. and Skinner C. 1990 Science $247,1553$.

Suckewer S. 1990 2nd International Colloguium, X-Ray Lasers, this proceedings.

Susskind S, Valeo E.J., Oberman C.R., and Bernstein, I.B., 1991, to be published Phys. Rev. A

Tighe W., Nam C.H., Goldhar J., Meixler L., Robinson J., Valeo E., and Suckewer S., 1990, SPIE Vol 1229 , Femtosecond to Nanosecond HighIntensity Lasers and Applications, 29

Trebes J. E. 1990 2nd International Colloquium, $X$-Ray Lasers, this proceedings. 